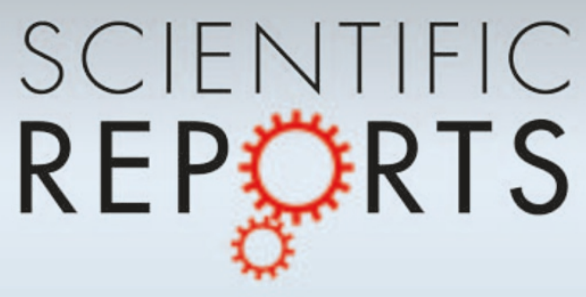

OPEN

SUBJECT AREAS:

MAGNETIC MATERIALS

AND DEVICES

APPLIED PHYSICS

MATERIALS PHYSICS

ELECTRONIC MATERIALS AND

DEVICES

Received

24 October 2011

Accepted

23 January 2012

Published

10 February 2012

Correspondence and requests for materials should be addressed to

S.V.D. (sebastiaan. van.dijken@aalto.fi)

\section{Electric-field control of magnetic domain wall motion and local magnetization} reversal

\author{
Tuomas H. E. Lahtinen, Kévin J. A. Franke \& Sebastiaan van Dijken
}

NanoSpin, Department of Applied Physics, Aalto University School of Science, P.O. Box 15100, Fl-00076 Aalto, Finland.

Spintronic devices currently rely on magnetic switching or controlled motion of domain walls by an external magnetic field or spin-polarized current. Achieving the same degree of magnetic controllability using an electric field has potential advantages including enhanced functionality and low power consumption. Here we report on an approach to electrically control local magnetic properties, including the writing and erasure of regular ferromagnetic domain patterns and the motion of magnetic domain walls, in $\mathrm{CoFe}-\mathrm{BaTiO}_{3}$ heterostructures. Our method is based on recurrent strain transfer from ferroelastic domains in ferroelectric media to continuous magnetostrictive films with negligible magnetocrystalline anisotropy. Optical polarization microscopy of both ferromagnetic and ferroelectric domain structures reveals that domain correlations and strong inter-ferroic domain wall pinning persist in an applied electric field. This leads to an unprecedented electric controllability over the ferromagnetic microstructure, an accomplishment that produces giant magnetoelectric coupling effects and opens the way to electric-field driven spintronics.

$\mathrm{n}$ the field of spintronics, researchers have controlled magnetic switching and domain wall motion using spinpolarized currents and these phenomena have formed the basis for magnetic random access memory $(\mathrm{MRAM})^{1}$ and magnetic nanowire device concepts $\mathrm{s}^{2,3}$. Employing significant amounts of electrical current, however, is inevitably accompanied by energy dissipation, and in this context, electric-field induced magnetization reversal without major current flow would be desirable. Multiferroic materials that combine ferromagnetism and ferroelectricity are a promising candidate for the realization of electric-field controlled spintronic devices ${ }^{4,5}$. However, practical requirements such as strong magnetoelectric coupling between both ferroic order parameters and room temperature operation pose significant scientific challenges for single-phase materials. Cross-linking between ferromagnetic and ferroelectric properties via interface effects in multiferroic heterostructures provides a viable alternative, as it allows for an independent optimization of both ferroic phases ${ }^{6}$. Up to now, several mechanisms for magnetoelectric coupling at ferromagnetic-ferroelectric interfaces have been explored including charge modulation ${ }^{7-14}$, exchange interactions ${ }^{15-21}$, and strain transfer ${ }^{22-30}$. This has led to great advances in electric-field control of macroscopic magnetic properties (anisotropy, exchange bias, and ferromagnetic resonance frequency) and the demonstration of interface-induced multiferroicity ${ }^{14}$. An extension of this degree of controllability to micromagnetic elements such as magnetic domains and domain walls would constitute another important step towards the realization of practical devices, as it would enable electric-field actuation of magnetic functions that up to now have only be addressed by an external magnetic field or spin-polarized current.

In this work we demonstrate that it is possible to precisely write and erase regular ferromagnetic domain patterns and to control the motion of magnetic domain walls in small electric fields. Our approach consists of two steps. First, a well-defined ferroelastic domain structure is fully transferred from a tetragonal ferroelectric to a magnetostrictive medium via interface strain transfer during thin film growth. Hereafter, an electrical bias voltage is used to reversibly manipulate the original ferromagnetic microstructure by polarization reversal and domain wall motion in the ferroelectric. The results show that microscopic features such as ferroelastic domain history and ferromagnetic-ferroelectric domain wall pinning critically determine the magnitude of macroscopic magnetoelectric coupling effects. This report is an extension of our previous work that focused on domain pattern transfer in multiferroic heterostructures ${ }^{31}$. Here, we for the first time explain the development of local magnetoelastic anisotropies in an applied electric field, we demonstrate electrical writing of regular ferromagnetic domain patterns, and we show that strong pinning between ferromagnetic domain walls and ferroelastic domain boundaries enables electric-field induced motion of magnetic domain walls. 
For this study, thin ferromagnetic CoFe films were directly grown onto ferroelectric $\mathrm{BaTiO}_{3}$ substrates with a regular ferroelastic stripe pattern that is typified by $90^{\circ}$ in-plane rotations of the ferroelectric polarization and elongated $c$ axis (Fig. 1a). This ferroelastic $a_{1}-a_{2}$ domain structure thus consisted of stripes wherein the elongation of the unit cell in the substrate film plane ( $a=3.992 \AA, c=4.036 \AA$ ) induced uniaxial lattice strain and magnetoelastic anisotropy in the overlaying ferromagnetic film. For full strain transfer, the relative difference in strain along the $c$ axis and $a$ axis in the ferromagnetic film equals $(c-a) / a=1.1 \%$. Rotation of the elongated $c$ axis by $90^{\circ}$ at the domain boundaries also realigns the uniaxial magnetoelastic anisotropy axis and, as a result, the magnetization direction is modulated by the ferroelectric stripe pattern. CoFe with an atomic percentage of $60 \%$ Co and $40 \%$ Fe was selected as ferromagnetic material because of its large magnetostriction and small magnetocrystalline anisotropy $^{32}$. This materials choice ensured that local magnetoelastic energies compared favorably to other energy scales within the ferromagnet (exchange, magnetostatic, and magnetocrystalline anisotropy energies), which is a prerequisite for electric-field control of ferromagnetic microstructures. Both ferroelectric and ferromagnetic domain patterns were imaged using optical polarization microscopy techniques. Real-time measurements of domain correlations in an applied electric field were facilitated by the use of optically semitransparent CoFe films with a thickness of $15 \mathrm{~nm}$. Partial optical reflection from both the $\mathrm{BaTiO}_{3}$ substrate and the CoFe film allowed for the imaging of ferroelectric (birefringent contrast) and ferromagnetic (magneto-optical Kerr effect contrast) domains in exactly the same sample area. More details on sample fabrication and characterization procedures are presented in the Methods section.

\section{Results}

Ferromagnetic-ferroelectric domain correlations. Full imprinting of ferroelastic $\mathrm{BaTiO}_{3}$ domains into CoFe during thin film growth is demonstrated in Fig. 1b. We find exact correlations between both ferroic domain patterns across the entire sample area. Analysis of the birefringent contrast of the ferroelectric substrate (FE) and the magneto-optical Kerr contrast of the ferromagnetic film (FM) indicates that the ferroelectric polarization and magnetization directions are collinear in zero applied magnetic field. Precise reproduction of the ferroelastic domain structure in the CoFe film is explained by strain transfer at the heterostructure interface, which induces local modulations in the uniaxial magnetoelastic anisotropy via inverse magnetostriction. The micromagnetic structure changes when an out-of-plane electric field of $E=10 \mathrm{kV} / \mathrm{cm}$ is applied across the $\mathrm{BaTiO}_{3}$ substrate. Figure 1d compares the ferroelectric and ferromagnetic domain patterns after reverting back to electrical remanence $(E=0 \mathrm{kV} / \mathrm{cm})$. The images provide clear evidence of highly correlated changes in both ferroic domain structures. In the $\mathrm{BaTiO}_{3}$ substrate, the electric field transforms the ferroelectric microstructure into alternating $a_{1}$ and $c$ domains with in-plane and out-of-plane polarization, respectively. These ferroelastic modifications are accompanied by the transfer of local strains to the CoFe film, which, in turn, results in the erasure (on top of $a_{1}$ domains) and conservation (on top of $c$ domains) of the original ferromagnetic stripe pattern. The schematic diagrams in Fig. 1 illustrate the correlations between the two ferroic microstructures in the as-deposited and electric-field induced states.

Imprinting of local magnetic anisotropies. To interpret the evolution of the ferromagnetic domain pattern, we need to first unravel the strain-mediated relationship between local magnetic anisotropies and the underlying ferroelectric domain structure. For this purpose, we utilized our polarization microscope to measure local magnetic hysteresis curves on single domain areas. Data as a function of in-plane magnetic field angle were used to determine the orientation of the uniaxial magnetic easy axis for each domain type. Moreover, the magnitude of the magnetoelastic anisotropy $\left(K_{m e}\right)$ was extracted by fitting the slope of hard-axis magnetization curves to $M_{s} / 2 K_{m e}$, where $M_{s}$ is the saturation magnetization of CoFe $(1.7 \times$ $\left.10^{6} \mathrm{~A} / \mathrm{m}\right)$. Figure 2 summarizes the results for all ferromagneticferroelectric domain combinations.

After growth, the ferromagnetic microstructure consists of alternating $a_{1}$ and $a_{2}$ domains with horizontal and vertical magnetic easy axes and $K_{m e}=1.7 \times 10^{4} \mathrm{~J} / \mathrm{m}^{3}$. This anisotropy value can be put into perspective by comparing it to a theoretical estimation. For polycrystalline films, $K_{m e}=3 \sigma \lambda / 2$, where $\lambda$ is the magnetostriction constant and $\sigma$ is the stress, which is proportional to the strain $\varepsilon$ via Young's modulus $Y$. Using $\varepsilon=1.1 \%$ for full strain transfer from the $\mathrm{BaTiO}_{3}$ substrate to the CoFe film and $\lambda=6.8 \times 10^{-532}$ and $Y=2.5 \times 10^{11}$ $\mathrm{J} / \mathrm{m}^{3}{ }^{33}$ for CoFe, gives $K_{\operatorname{me}}$, max $=2.8 \times 10^{5} \mathrm{~J} / \mathrm{m}^{3}$. This calculation suggests that less than $10 \%$ of the $\mathrm{BaTiO}_{3}$ lattice strain is transferred during $\mathrm{CoFe}$ film growth. Yet, our experiments clearly demonstrate a

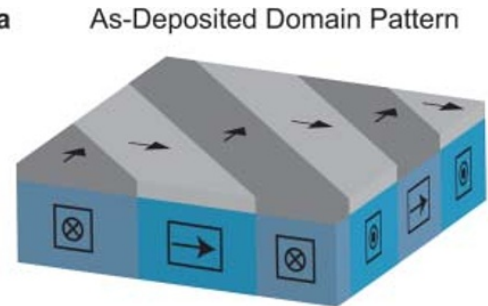

c Electric-Field Induced Domain Pattern

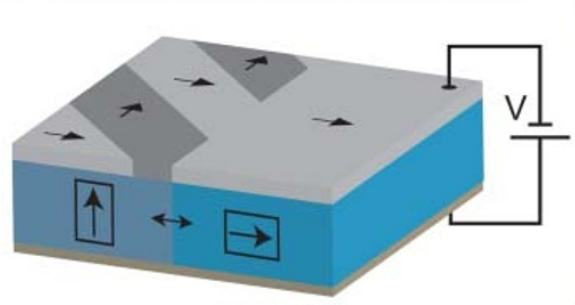

b
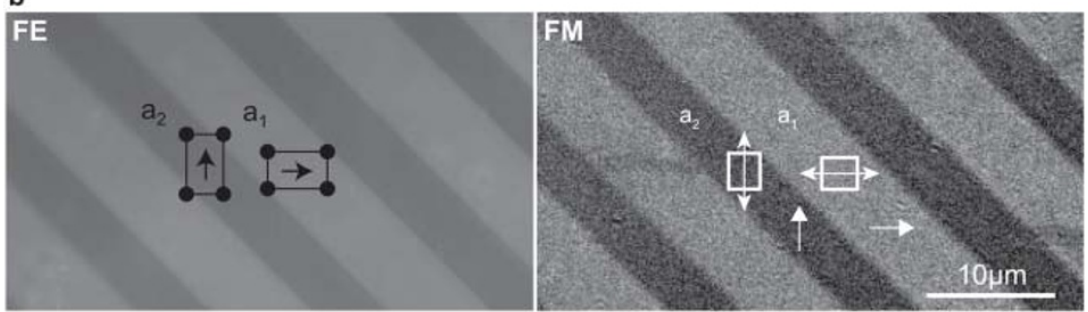

d
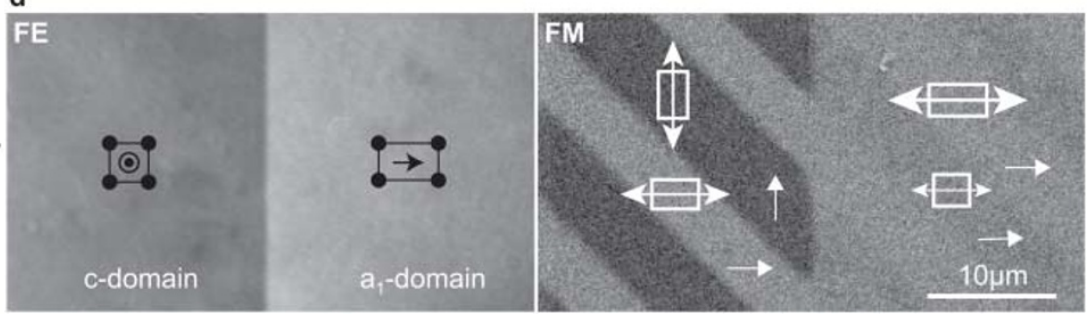

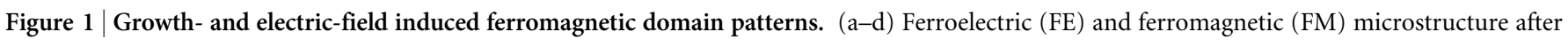

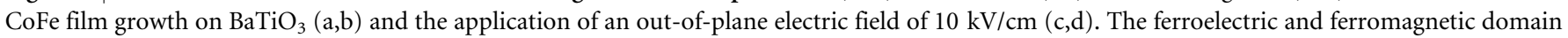

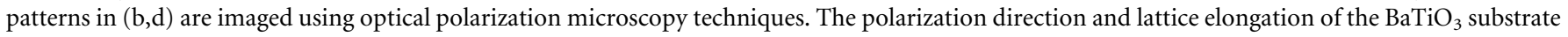
(black rectangles with arrows), the orientation of the strain-induced magnetic easy axis (white rectangles with double-headed arrows), and the magnetization direction in zero applied magnetic field (white arrows) are indicated. 


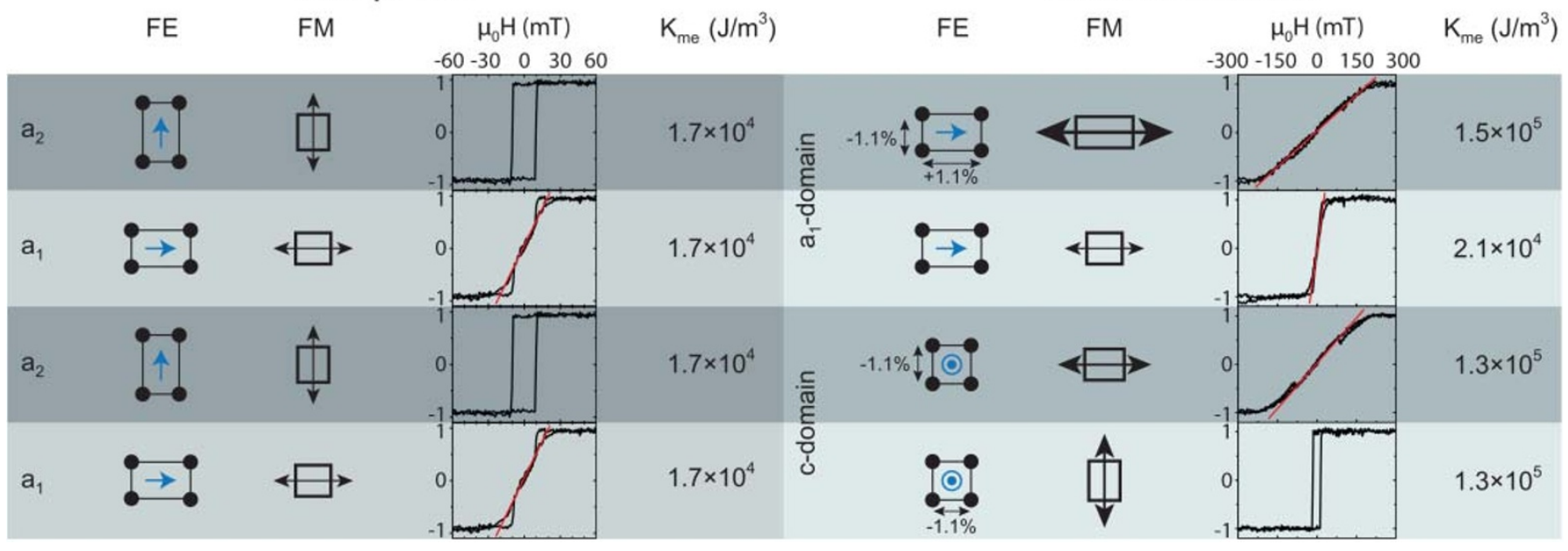

Figure $2 \mid$ Relation between local magnetic anisotropies in the $\mathrm{CoFe}$ film and the ferroelectric domain structure of the $\mathrm{BaTiO}_{3}$ substrate. The hysteresis curves are measured on single domain areas with the applied in-plane magnetic field along the vertical axis of all images in this article. The solid red lines are linear fits to the hard-axis magnetization curves.

that such limited strain effect is sufficient to fully imprint ferroelastic domains into magnetostrictive films when other anisotropy contributions are small.

Figure $1 \mathrm{~d}$ shows that the ferromagnetic microstructure consists of four different domain states after the application of an out-of-plane electric field. On top of ferroelectric $a_{1}$ domains, new compressive and tensile strains in the original $a_{2}$ stripes of the CoFe film forced the uniaxial magnetic easy axis to rotate by $90^{\circ}$. As a result, the easy magnetization directions of neighboring magnetic stripes align parallel and, thus, the growth-induced domain pattern is electrically erased. The magnitude of the uniaxial magnetic anisotropy in the original $a_{2}$ stripes is found to increase significantly to $K_{m e}=1.5 \times$ $10^{5} \mathrm{~J} / \mathrm{m}^{3}$. This value compares more favorably to the theoretical $K_{m e, \max }$ and it clearly indicates that strain transfer is more efficient when the CoFe film is clamped to the $\mathrm{BaTiO}_{3}$ substrate (as compared to the initial stages of film growth). For the magnetic $a_{1}$ stripes, the underlying ferroelectric lattice symmetry did not change, hence, $K_{m e}$ remains small $\left(2.1 \times 10^{4} \mathrm{~J} / \mathrm{m}^{3}\right)$.

Similar arguments hold for the development of magnetic anisotropies on top of out-of-plane ferroelectric $c$ domains. In this case, the ferroelectric domain exhibits cubic crystal lattice symmetry in the substrate plane. Consequently, the CoFe film is compressed along the same axis along which it obtained a small tensile strain during deposition. As the electric-field controlled lattice compression in the CoFe film exceeds the growth-induced strain, the uniaxial magnetic easy axis rotates by $90^{\circ}$ in both stripe domains and the strength of the magnetic anisotropy is found to increase to $K_{m e}=1.3 \times 10^{5} \mathrm{~J} / \mathrm{m}^{3}$. The original magnetic $a_{1}$ and $a_{2}$ domains thus transform to $a_{2}$ and $a_{1}$ stripes, respectively, but the overall pattern remains the same. Electric-field induced formation of ferroelectric $c$ domains thus results in the conservation and stabilization of the original ferromagnetic domain structure.

Electric-field writing of ferromagnetic domain patterns. We now demonstrate that strain-mediated ferroic domain correlations in $\mathrm{CoFe}-\mathrm{BaTiO}_{3}$ can be used to write and erase regular ferromagnetic domain patterns in small electric fields. An example is shown in Fig. 3, which depicts the evolution of the ferromagnetic domain structure as a function of out-of-plane bias voltage. First, the ferroelectric polarization of the $\mathrm{BaTiO}_{3}$ substrate is saturated by a bias voltage of $120 \mathrm{~V}$ (Fig. 3a). As previously discussed, this uniform $c$ domain produces a regular ferromagnetic stripe pattern in the $\mathrm{CoFe}$ film. Reverting back to zero bias causes the ferroelectric microstructure to relax into alternating $a_{1}$ and $c$ domains (Fig. 3b).
Consequently, the local strain state in the CoFe film and, thereby, the magnetoelastic anisotropies alter in accordance with Fig. 2. This results in the erasure of magnetic stripes on top of the ferroelectric $a_{1}$ domains. Subsequent application of a small bias voltage causes the

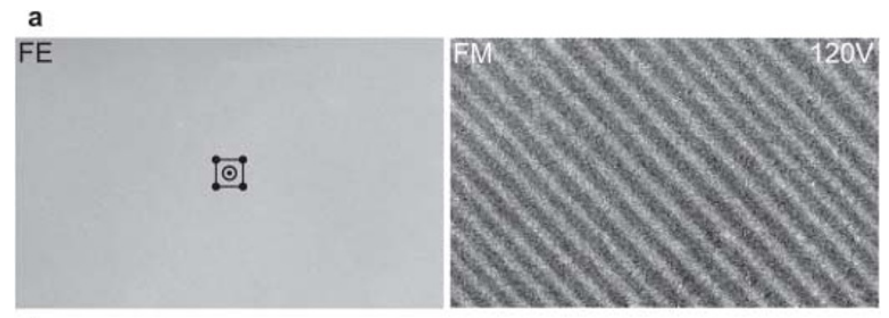

b
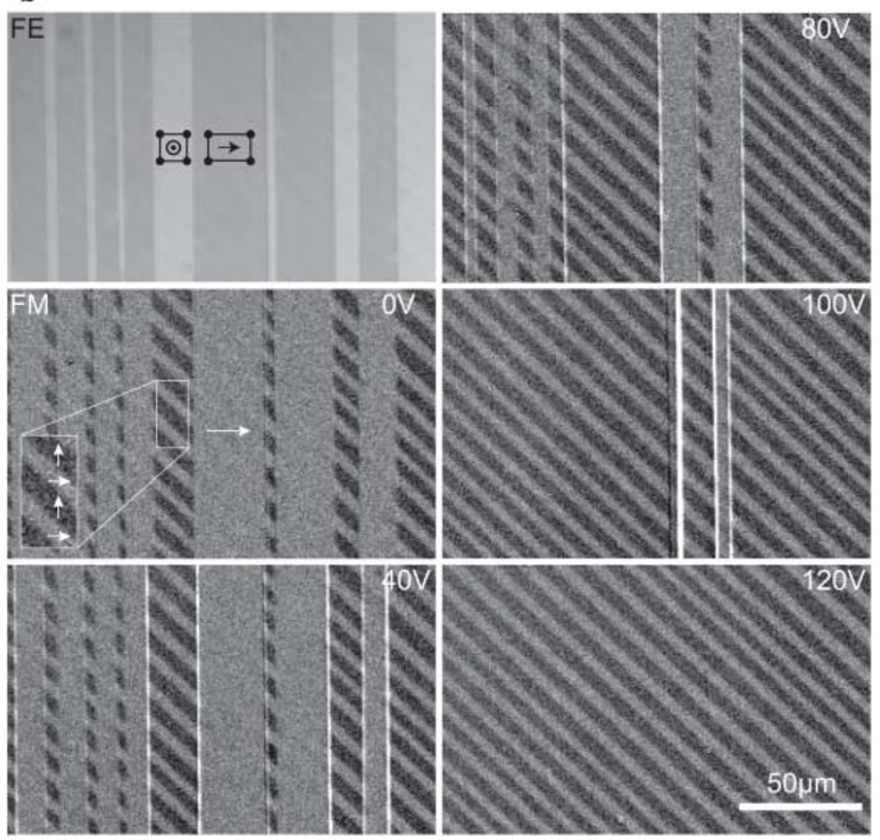

Figure 3 Recurrent electric-field writing and erasure of ferromagnetic domain patterns. (a,b) Polarization microscopy images of the ferroelectric (FE) and ferromagnetic (FM) domain structure during the application of an out-of-plane bias voltage. Correlations between the ferromagnetic microstructure and the underlying ferroelectric lattice are evident. 
ferroelectric $c$ domains to grow at the expense of $a_{1}$ domains by sideways motion of the ferroelastic $a_{1}-c$ domain boundaries. This again modifies the local strain states in the CoFe film and the regular magnetic stripe pattern is electrically rewritten. The images of Fig. 3 indicate that electric writing and erasure of ferromagnetic patterns based on strain-mediated correlations between local magnetic anisotropies and underlying ferroelastic domains is reversible. We note that this unprecedented electric-field control of ferromagnetic microstructures was obtained at room temperature without the application of a magnetic field.

Macroscopically, interlinking between ferromagnetic and ferroelectric order parameters is often quantified by the converse magnetoelectric coupling coefficient, $\alpha=\mu_{0} \Delta M / \Delta E$. In our study, this coefficient is estimated from the electric-field induced change in the sample area that is covered by ferroelectric $a_{1}$ domains $\left(\Delta a_{1}\right)$. At $0 \mathrm{~V}$, the ferroelectric $a_{1}$ domains cover about $65 \%$ of the CoFe film and this reduces to $0 \%$ at $120 \mathrm{~V}(E=2.4 \mathrm{kV} / \mathrm{cm})$. By also taking into account that half of the CoFe film magnetization on top of the $\mathrm{BaTiO}_{3}$ substrate rotates by $90^{\circ}$ when the ferroelectric $a_{1}$ domains are replaced by ferroelectric $c$ domains, we find $\alpha=0.5 \mu_{0} M_{s} \Delta a_{1} / \Delta E$ $=3 \times 10^{-6} \mathrm{~s} / \mathrm{m}$, which is about one order of magnitude larger than previously reported results ${ }^{23,29}$. Our experiments thus clearly indicate that giant magnetoelectric coupling effects can be realized by careful optimization of the ferromagnetic and ferroelectric microstructures.

Electric-field induced magnetic domain wall motion. Another intriguing observation in our work is electric-field control of magnetic domain wall motion. Here we analyze the main effects and the underlying driving mechanism. Figure $4 \mathrm{a}$ shows an enlarged image of the ferromagnetic domain pattern on top of ferroelectric $c$ and $a_{1}$ domains. Two different types of magnetic domain walls are distinguished. The walls labeled as (1), appear on top of ferroelectric $c$ domains and their location is determined by the position of $a_{1}-a_{2}$ boundaries in the $\mathrm{BaTiO}_{3}$ substrate during CoFe film growth. Thus, although this type of magnetic wall is not directly pinned onto an existing ferroelastric boundary, its physical properties (location, width, and pinning strength) are determined by local magnetic anisotropies that result from a previous ferroelastic domain configuration. This observation underpins the importance of ferroelastic domain history on magnetoelectric coupling effects. The length of this type of magnetic domain wall equals $\sqrt{2}$ times the width of the ferroelectric $c$ domains and, hence, these walls extend over the entire sample in saturation $(120 \mathrm{~V})$. In contrast, the magnetic domain walls labeled as (2) in Fig. 4a are pinned on top of existing ferroelastic $a_{1}-c$ domain boundaries. Sideways motion of such ferroelastic boundaries in an out-of-plane electric field results in the reappearance of type- 1 walls at pre-defined locations, while type2 walls are dragged along by their ferroelectric counterpart (Fig. 3). The length of type- 2 walls varies from $4 \mathrm{~nm}$ to $10 \mathrm{~nm}$, which is $\sqrt{2}$ times the original $a_{1}-a_{2}$ stripe width. To analyze the properties of magnetic domain walls in more detail, we performed micromagnetic simulations using the local magnetic anisotropies of Fig. 2 as input parameters (Fig. 4b). From these simulations, the spin rotation within the domain wall and the pinning energy are extracted. The results confirm that type- 1 and type- 2 domain walls are strongly pinned onto previous and existing ferroelastic boundaries, respectively (Fig. 4c-d). The pinning energy profile provides an ever increasing barrier for lateral wall displacement which cannot be overcome by thermal activation effects. Pinning of ferromagnetic domain walls onto ferroelastic boundaries is therefore robust and the strong link between both ferroic domain walls is only broken if one of the walls is destroyed by saturation of its ferromagnetic or ferroelectric order. These results show that it is possible to accurately control the position of pinned magnetic domain walls by sideways motion of ferroelastic boundaries in an applied electric field.

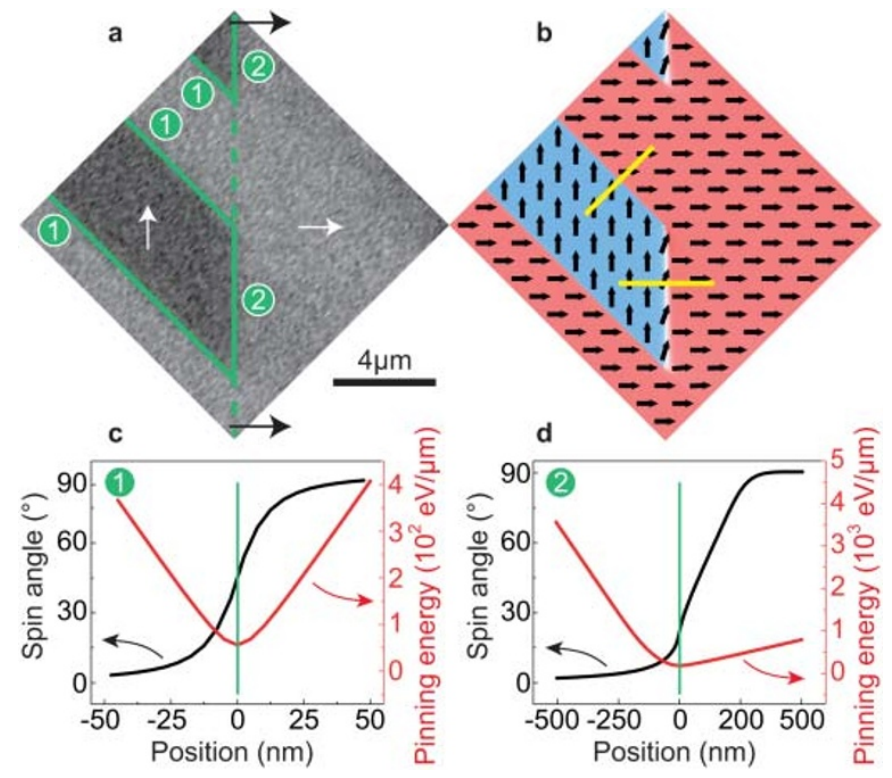

Figure 4 | Electric-field induced magnetic domain wall motion.

(a) Polarization microscopy image of the ferromagnetic domain structure on top of ferroelectric $c$ (left) and $a_{1}$ (right) domains. Two types of magnetic domain walls (numbered by (1) and (2)) and the underlying $a_{1}-$ $c$ domain boundary are indicated by solid and dashed lines, respectively. The black arrows indicate the direction of domain wall motion in an outof-plane electric field. (b) Micromagnetic simulation of the magnetic microstructure in (a). (c,d) Spin rotation within the magnetic domain walls and pinning energies indicating strong clamping of ferromagnetic walls onto ferroelastic boundaries (or magnetic anisotropy distributions that are the results of such boundaries). The pinning energy is calculated per $\mu \mathrm{m}$ domain wall length. The asymmetry in the wall profile and pinning energy in (d) is due to different magnetic anisotropy strengths on the left $\left(K_{m e}=1.3 \times 10^{5} \mathrm{~J} / \mathrm{m}^{3}\right)$ and right $\left(K_{m e}=2.1 \times 10^{4} \mathrm{~J} / \mathrm{m}^{3}\right)$ side of the ferroelastic domain boundary.

\section{Discussion}

We have demonstrated that strain transfer from ferroelastic domains in ferroelectric media to magnetostrictive materials can be used to exactly copy ferroelectric domain patterns into ferromagnetic thin films. This provides us with an unprecedented ability to write and erase ferromagnetic domains in an electric field and to accurately control the motion of magnetic domain walls. Polarization microscopy analysis of both ferroic domain structures after thin film growth and during the application of an electric field reveals that a strain-mediated mechanism couples the ferroelectric and ferromagnetic microstructures. The efficiency of lattice strain transfer during film growth is found to be less than $10 \%$. Yet, by selecting a ferromagnetic material that combines large magnetostriction and negligible magneto-crystalline anisotropy it is possible to obtain full coherency between ferroelectric and ferromagnetic domains. Another important observation is the persistence of growth-induced ferromagnetic domain patterns. When the ferroelectric microstructure is altered by the application of an electric field, new ferroelectric domains are imprinted into the ferromagnetic film by efficient strain transfer. However, the original ferromagnetic stripe domains that are formed during film growth remain visible. The new ferromagnetic microstructure is a superposition of two domain patterns and consists of four domains with distinctive uniaxial magnetoelastic anisotropies (Fig. 2). The observation of this memory effect has important implications for electric-field control of ferromagnetic domains and, more generally, 
for magnetoelectric coupling between ferromagnetic and ferroelectric order parameters. In carefully designed heterostructures it can be used to electrically write and erase well-defined ferromagnetic domain patterns over large areas and to establish giant magnetoelectric coupling effects. Another consequence of ferroelastic domain history is the formation of ferromagnetic domain walls (type-1) at pre-defined locations in an applied electric field.

Pinning of ferromagnetic domain walls on top of ferroelastic boundaries enables electric-field control of magnetic domain wall motion. This is an interesting finding because it offers a low-power alternative to spin-torque driven domain wall dynamics, which requires the passing of spin-polarized currents. In our $\mathrm{CoFe}$ $\mathrm{BaTiO}_{3}$ heterostructures, strong coupling between ferromagnetic and ferroelastic domain walls is due to an abrupt rotation of the strain-induced magnetoelastic anisotropy axis at $90^{\circ}$ ferroelastic boundaries. Micromagnetic simulations indicate that the pinning strength is proportional to the magnetoelastic anisotropy constant $\left(K_{m e}\right)$ and that the energy profile increases linearly for large magnetic wall displacements away from ferroelastic boundaries. As a result, the magnitude of the potential barrier cannot be overcome for sufficiently large domain wall separations. This provides us with the unique ability to accurately control the position of ferromagnetic domain walls by sideways motion of the underlying ferroelastic boundaries in an applied electric field. We note that these promising results represent a proof-of-concept and that future experimental and theoretical studies are necessary to carefully examine the dynamics of coupled ferroic domain walls. Also, we expect that ferromagnetic domain wall pinning onto ferroelastic boundaries is a generic effect and that these results are transferrable to patterned nanostructures and other electric field and ferroelastic domain configurations.

\section{Methods}

Thin CoFe films with an atomic percentage of $60 \%$ Co and $40 \%$ Fe were directly grown onto ferroelectric $\mathrm{BaTiO}_{3}$ substrates by electron-beam evaporation at room temperature. Each film was covered by a $3 \mathrm{~nm}$ thick Au capping layer to prevent oxidation under atmospheric conditions. The thickness of the CoFe films was $15 \mathrm{~nm}$ and $\mathrm{x}$-ray diffraction, atomic force microscopy, and transmission electron microscopy measurements confirmed the growth of smooth polycrystalline layers with a small root mean square roughness of $<0.5 \mathrm{~nm}$. The $0.5 \mathrm{~mm}$ thick $\mathrm{BaTiO}_{3}$ substrates contained a regular $a_{1}-a_{2}$ domain stripe pattern during CoFe film growth. The width of the ferroelastic stripes varied from $3-7 \mu \mathrm{m}$.

The ferromagnetic and ferroelectric domains were imaged independently using optical polarization microscopy techniques. The microscope consisted of polarizing optics, an adjustable diaphragm, a $100 \times$ objective, a CCD camera and an electromagnet for the application of in-plane magnetic fields. The ferroelectric $\mathrm{BaTiO}_{3}$ domains were imaged using birefringent contrast with the polarizer and analyzer in cross-configuration. During these measurements the CoFe film was saturated by an in-plane magnetic field to eliminate magneto-optical Kerr contrast from overlying magnetic domains. The magnetic domain patterns were measured using the magneto-optical Kerr effect. In this case, the polarizing optics was set to a few degrees from extinction (to optimize the magneto-optical Kerr contrast) and a background subtraction method was used to zero-out contrast from structural defects and ferroelectric domains. Local magnetic hysteresis curves were constructed from the evolution of magnetic contrast in single domain areas during magnetization reversal. All ferromagnetic domain images in this paper (including those recorded as a function of out-of-plane bias voltage) were obtained at room temperature and zero applied magnetic field. Electric fields were generated by using the $\mathrm{CoFe} / \mathrm{Au}$ film as top electrode and silver paint on the back of the $\mathrm{BaTiO}_{3}$ substrates as bottom electrode. The electric bias voltage was ramped at $10-20 \mathrm{~V} / \mathrm{minute}$ and a $20 \times$ objective was used to provide enough space for wiring during the poling experiment (Fig. 3).

The micromagnetic simulations (Fig. 4) were conducted using object oriented micromagnetic framework (OOMMF) software ${ }^{34}$. To closely mimic the experiments, the local magnetoelastic anisotropies of Fig. 2 were used as input parameter for the different magnetic domains. Other parameters included a saturation magnetization of $M_{s}=1.7 \times 10^{6} \mathrm{~A} / \mathrm{m}$ and a uniform exchange constant of $K_{e x}=2.1 \times 10^{-11} \mathrm{~J} / \mathrm{m}$. The domain wall pinning potentials were calculated from the orientation of magnetic spins along a line perpendicular to the walls using $U(x)=l t \int^{\infty} K_{m e}(x) \sin ^{2}(\theta(x)-\alpha(x)) \mathrm{d} x$, where $K_{m e}(x), \theta(x)$, and $\alpha(x)$ are the strain-induced magnetoeleastic anisotropy, the angle of the spin moment, and the orientation of the uniaxial magnetic easy axis as a function of position, respectively. The parameters $l$ and $t$ indicate the length of the domain wall and the film thickness.
In Figs. $4 \mathrm{c}$ and $4 \mathrm{~d}$, the pinning potential is calculated per $\mu \mathrm{m}$ domain wall length, i.e. $l=1 \mu \mathrm{m}$.

1. Chappert, C., Fert, A. \& Nguyen van Dau, F. The emergence of spin electronics in data storage. Nature Mater. 6, 813-823 (2007).

2. Allwood, D. A., Xiong, G., Faulkner, C. C., Atkinson, D., Petit, D. \& Cowburn, R. P. Magnetic domain-wall logic. Science 309, 1688-1692 (2005).

3. Parkin, S. S. P., Hayashi, M. \& Thomas, L. Magnetic domain-wall racetrack memory. Science 320, 190-194 (2008).

4. Eerenstein, W., Mathur, N. D. \& Scott, J. F. Multiferroic and magnetoelectric materials. Nature 442, 759-765 (2006).

5. Ramesh, R. \& Spaldin, N. A. Multiferroics: progress and prospects in thin films. Nature Mater. 6, 21-29 (2007).

6. Ma, J., Hu, J., Li, Z. \& Nan, C-W. Recent progress in multiferroic magnetoelectric composites: from bulk to thin films. Adv. Mater. 23, 1062-1087 (2011).

7. Duan, C-G., Jaswal, S. S. \& Tsymbal, E. Y. Predicted magnetoelectric effect in $\mathrm{Fe} / \mathrm{BaTiO}_{3}$ multilayers: ferroelectric control of magnetism. Phys. Rev. Lett. 97, 047201 (2006).

8. Weisheit, M., Fähler, S., Marty, A., Souche, Y., Poinsignon, C. \& Givord, D. Electric field-induced modification of magnetism in thin-film ferromagnets. Science 315, 349-351 (2007).

9. Duan, C-G., Velev, J. P., Sabirianov, R. F., Mei, W. N., Jaswal, S. S. \& Tsymbal, E. Y. Tailoring magnetic anisotropy at the ferromagnetic/ferroelectric interface. Appl. Phys. Lett. 92, 122905 (2008).

10. Fechner, M. et al. Magnetic phase transition in two-phase multiferroics predicted from first principles. Phys. Rev. B 78, 212406 (2008).

11. Molegraaf, H. J. A. et al. Magnetoelectric effects in complex oxides with competing ground states. Adv. Mater. 21, 2470-2474 (2009).

12. Garcia, V. et al. Ferroelectric control of spin polarization. Science 327, 1106-1110 (2010).

13. Lee, J., Sai, N., Cai, T., Niu, Q. \& Demkov, A. A. Interfacial magnetoelectric coupling in tricomponent superlattices. Phys. Rev. B 81, 144425 (2010).

14. Valencia, S. et al. Interface-induced room-temperature multiferroicity in $\mathrm{BaTiO}_{3}$ Nature Mater. 10, 753-758 (2011)

15. Borisov, P., Hochstrat, A., Chen, X., Kleemann, W. \& Binek, C. Magnetoelectric switching of exchange bias. Phys. Rev. Lett. 94, 117203 (2005).

16. Laukhin, V. et al. Electric-field control of exchange bias in multiferroic epitaxial heterostructures. Phys. Rev. Lett. 97, 227201 (2006).

17. Chu, Y-H. et al. Electric-field control of local ferromagnetism using a magnetoelectric multiferroic. Nature Mater. 7, 478-482 (2008).

18. Béa, H. et al. Mechanisms of exchange bias with multiferroic $\mathrm{BiFeO}_{3}$ epitaxial thin films, Phys. Rev. Lett. 100, 017204 (2008).

19. Lebeugle, D., Mougin, A., Viret, M., Colson, D. \& Ranno, L. Electric field switching of the magnetic anisotropy of a ferromagnetic layer exchange coupled to the multiferroic compound $\mathrm{BiFeO}_{3}$. Phys. Rev. Lett. 103, 257601 (2009).

20. He, X. et al. Robust isothermal electric control of exchange bias at room temperature. Nature Mater. 9, 579-585 (2010).

21. Wu, S. M. et al. Reversible electric control of exchange bias in a multiferroic fieldeffect device. Nature Mater. 9, 756-761 (2010).

22. Thiele, C., Dörr, K., Bilani, O., Rödel, J. \& Schultz, L. Influence of strain on the magnetoelectric effect in $\mathrm{La}_{0.7} \mathrm{~A}_{0.3} \mathrm{MnO}_{3} / \mathrm{PMN}-\mathrm{PT}(001)(\mathrm{A}=\mathrm{Sr}, \mathrm{Ca})$. Phys. Rev $B$ 75, 054408 (2007).

23. Eerenstein, W., Wiora, M., Prieto, J. L., Scott, J. F. \& Mathur, N. D. Giant sharp and persistent converse magnetoelectric effects in multiferroic epitaxial heterostructures. Nature Mater. 6, 348-351 (2007).

24. Sahoo, S., Polisetty, S., Duan, C-G., Sitaram, S., Jaswal, S. \& Tsymbal, E. Y. Ferroelectric control on magnetism in $\mathrm{BaTiO}_{3} / \mathrm{Fe}$ heterostructures via interface strain coupling. Phys. Rev. B 76, 092108 (2007).

25. Brandlmaier, A. et al. In situ manipulation of magnetic anisotropy in magnetite thin films. Phys. Rev. B 77, 104445 (2008).

26. Lou, J., Liu, M., Reed, D., Ren, Y. \& Sun, N. X. Giant electric field tuning of magnetism in novel multiferroic FeGaB/lead zinc niobate-lead titanate (PZN-PT) heterostructures. Adv. Mater. 21, 4711-4715 (2009).

27. Dörr, K. et al. A model system for strain effects: epitaxial magnetic films on a piezoelectric substrate. Eur. Phys. J. B 71, 361-366 (2009).

28. Geprägs, S., Brandlmaier, A., Opel, M., Gross, R. \& Goennenwein, S. T. B. Electric field controlled manipulation of the magnetization in $\mathrm{Ni} / \mathrm{BaTiO} 3$ hybrid structures. Appl. Phys. Lett. 96, 142509 (2010).

29. Chen, Y., Fitchorov, T., Vittoria, C. \& Harris, V. G. Electrically controlled magnetization switching in a multiferroic heterostructure. Appl. Phys. Lett. 97, 052502 (2010).

30. Venkataiah, G., Shirahata, Y., Itoh, M. \& Taniyama, T. Manipulation of magnetic coercivity of $\mathrm{Fe}$ film in $\mathrm{Fe} / \mathrm{BaTiO}_{3}$ heterostructures by electric field. Appl. Phys. Lett. 99, 102506 (2011).

31. Lahtinen, T. H. E., Tuomi, J. O. \& van Dijken, S. Pattern transfer and electric-fieldinduced magnetic domain formation in multiferroic heterostructures. Adv. Mater. 23, 3187-3191 (2011).

32. Hall, R. C. Magnetic anisotropy and magnetostriction of ordered and disordered cobalt-iron alloys, J. Appl. Phys. 31, 157S-158S (1960). 
33. Vas'ko, V. A., Rantschler, J. O. \& Kief, M. T. Structure, stress, and magnetic properties of high saturation magnetization films of FeCo. IEEE Trans. Magn. 40, 2335-2337 (2004).

34. http://math.nist.gov/oommf.

\section{Acknowledgements}

The work was supported by the Academy of Finland under contract no. 127731. T.H.E.L. and K.J.A.F. also like to acknowledge support from the National Doctoral Program in Materials Physics and the Finnish Doctoral Program in Computational Sciences.

\section{Author contributions}

T.H.E.L and S.v.D. conceived and carried out the experiments. K.J.A.F. conducted the micromagnetic simulations. T.H.E.L, K.J.A.F and S.v.D. analyzed the data. T.H.E.L and S.v.D. wrote the manuscript.

\section{Additional information}

Competing financial interests: The authors declare no competing financial interests.

License: This work is licensed under a Creative Commons

Attribution-NonCommercial-ShareAlike 3.0 Unported License. To view

a copy of this license, visit http://creativecommons.org/licenses

/by-nc-sa/3.0/

How to cite this article: Lahtinen, T.H.E., Franke, K.J.A. \& van Dijken, S. Electric-field control of magnetic domain wall motion and local magnetization reversal. Sci. Rep. 2, 258; DOI:10.1038/srep00258 (2012) 